Bhely ANGoBoy ILONDEA $A^{1,2,3}$

Hans BEECKMAN ${ }^{2}$

Dakis-Yaoba OUÉDRAOGO ${ }^{4}$

Nils BOURLAND $2,5,6$

TOM DE MIL ${ }^{2}$

Jan VAN DEN BuLCKE ${ }^{3}$

Joris VAN ACKER ${ }^{3}$

Camille Couralet ${ }^{2,3}$

Corneille EWANGo ${ }^{7}$

Wannes HuBAU ${ }^{2}$

Benjamin ToIRAMBE ${ }^{8}$

Jean-Louis DouCET

Adeline FAYOLLE ${ }^{4}$

${ }^{1}$ Institut national pour l'étude et la recherche agronomiques Kinshasa

République démocratique du Congo

${ }^{2}$ Musée royal de l'Afrique centrale

Service de biologie du bois

Tervuren

Belgique

${ }^{3}$ Ghent University

UGCT-UGent-Woodlab

Laboratory of Wood Technology

Department of Environment

B-9000 Gand

Belgique

${ }^{4}$ Gembloux Agro-Bio Tech

Université de Liège

Passage des Déportés 2

5030 Gembloux

Belgique

${ }^{5}$ Centre de recherche forestière internationale (CIFOR)

Jalan CIFOR

Situ Gede, Sindang Barang

Bogor (Barat) 16115

Indonésie

${ }^{6}$ Resources \& Synergies Development Pte Ltd Singapour

${ }^{7}$ Université de Kisangani

Faculté de gestion des ressources naturelles Kisangani

République démocratique du Congo

${ }^{8}$ Ministère de l'Environnement

et Développement durable

Kinshasa

République démocratique du Congo

Auteur correspondant /

Corresponding author:

Bhely Angoboy Ilondea -

bhely.angoboy@gmail.com

\title{
Une forte saisonnalité du climat et de la phénologie reproductive dans la forêt du Mayombe : l'apport des données historiques de la Réserve de Luki en République démocratique du Congo
}

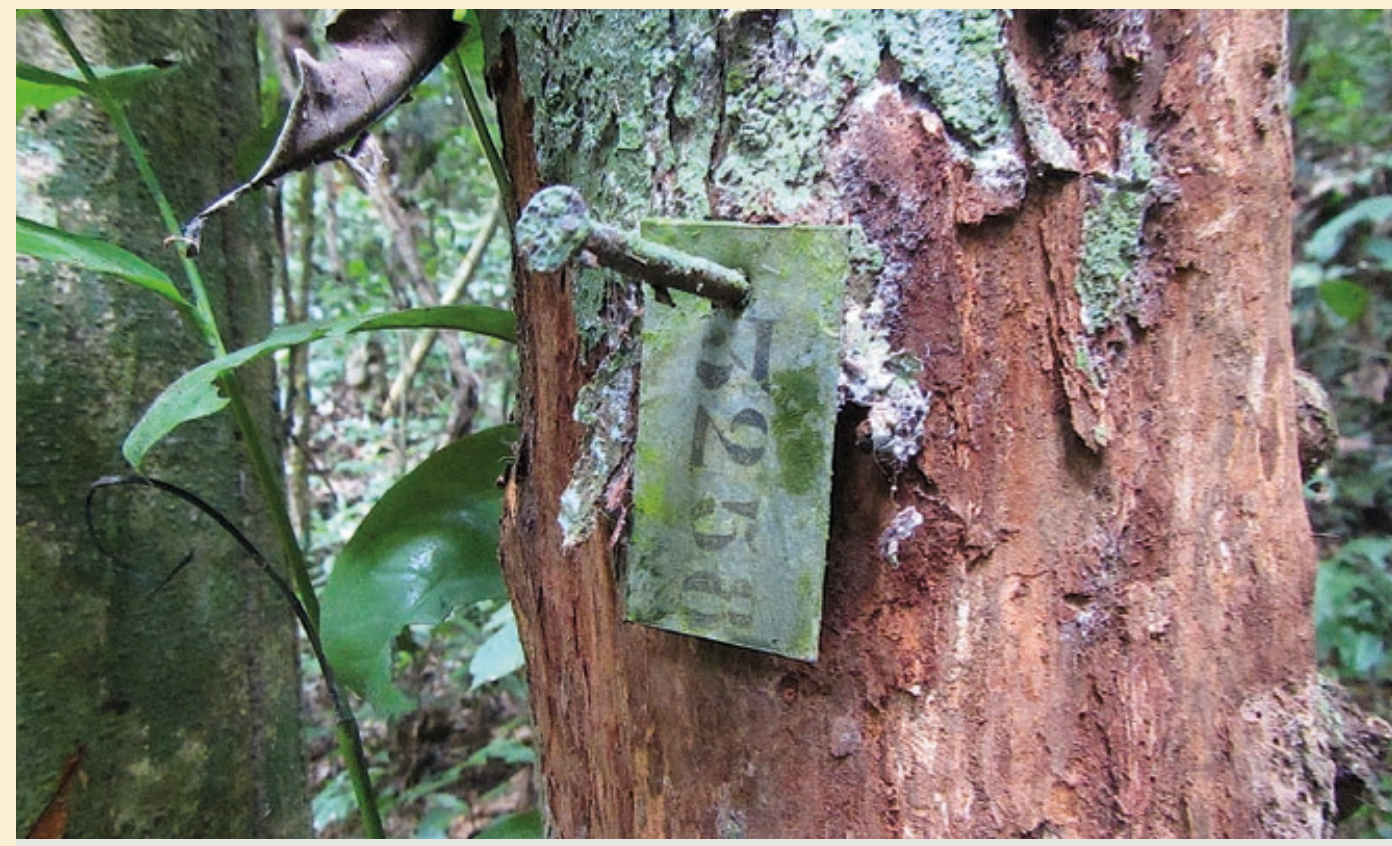

Photo 1.

Arbre marqué pour le suivi de la phénologie et de la croissance dans le parc de la Nkula. Photo B. Angoboy llondea.

Doi : 10.19182/bft2019.341.a31753 - Droit d'auteur @ 2019, Bois et Forêts des Tropiques - @ Cirad - Date de soumission : 10 septembre 2018 ;

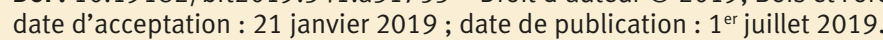

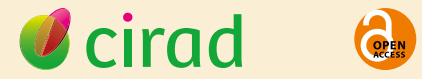

(c) $(1) \Theta$

Licence Creative Commons :

Attribution - Pas de Modification 4.0 International.

Attribution-NoDerivatives 4.0 International (CC BY-ND 4.0)
Citer l'article / To cite the article

Angoboy llondea B., Beeckman H., Ouédraogo D.-Y., Bourland N., De Mil T., Van Den Bulcke J., Van Acker J., Couralet C., Ewango C., Hubau W., Toirambe B., Doucet J.-L., Fayolle A., 2019. Une forte saisonnalité du climat et de la phénologie reproductive dans la forêt du Mayombe : l'apport des données historiques de la Réserve de Luki en République démocratique du Congo. Bois et Forêts des Tropiques, 341 : 39-53. Doi : https://doi.org/10.19182 bft2019.341.a31753 
B. Angoboy Ilondea, H. Beeckman, D.-Y. OUÉdRAOGo, N. BouRLANd, T. DE MIL, J. Van Den Bulcke, J. Van Acker, C. Couralet, C. Ewango, W. Hubau, B. Tolrambe, J.-L. Doucet, A. FAYolle

\section{RÉSUMÉ}

Une forte saisonnalité du climat et de la phénologie reproductive dans la forêt du Mayombe : l'apport des données historiques de la Réserve de Luki en République démocratique du Congo

En Afrique, la phénologie reproductive des arbres tropicaux, majoritairement annuelle, semble présenter une certaine régularité. Cette étude documente les variations intra- et interannuelles de la phénologie reproductive des arbres de la forêt du Mayombe à partir des données historiques de la Réserve de Luki (République démocratique du Congo). Le diamètre de reproduction des espèces exploitées pour le bois, encore largement méconnu, a été également examiné pour les espèces suffisamment représentées dans les données. Le suivi phénologique de 3642 arbres appartenant à 158 espèces et 39 familles a été réalisé tous les 10 jours de 1948 à 1957. Les statistiques circulaires ont été utilisées pour tester le synchronisme de la phénologie entre arbres, à l'échelle de la communauté, pour la forêt dans son ensemble, et individuellement pour 87 espèces, dont 35 espèces bien représentées ( $n \geq 20$ arbres), 16 espèces commerciales et 36 autres espèces. Des régressions logistiques ont permis de déterminer le diamètre de fructification (minimum et régulier) de ces espèces. Pour la majorité des espèces, la floraison était régulière, annuelle et largement saisonnière $(81,6 \%$, 71 espèces). Les pics étaient plus marqués pour la floraison que pour la fructification, plus étalée dans le temps, bien que significativement agrégée temporellement. La majorité des arbres et des espèces fleurissaient entre décembre et février, pendant la petite saison sèche, bien que des fleurs et des fruits étaient observables toute l'année à l'échelle de la communauté. Seules 13 espèces ont montré une relation significative entre le diamètre et la reproduction, parmi lesquelles sept espèces de canopée, cinq de sous-bois et une héliophile. Pour ces espèces, la moyenne du diamètre minimum de reproduction était de $17,3 \mathrm{~cm}$.

Mots-clés : diamètre de reproduction, floraison, fructification, forêts tropicales humides, forêt du Mayombe, saisonnalité, synchronisme, République démocratique du Congo.

\section{ABSTRACT}

\section{Highly seasonal climate and reproductive phenology in the Mayombe forest: contribution of historical data from the Luki Reserve in the Democratic Republic of Congo}

In Africa, the reproductive phenology of tropical trees is mostly annual and regular. This study documents the intra- and inter-annual reproductive phenology of trees in the Mayombe forest, from historical data on the Luki Reserve in the Democratic Republic of the Congo. Reproductive diameter, which is a key parameter for forest management but is largely unknown for many timber species, was also documented for the most abundant species in the dataset. Phenological monitoring of 3,642 trees belonging to 158 species and 39 families was conducted in the Luki Reserve every 10 days from 1948 to 1957. Circular statistics were used to test the synchronicity of phenological events among trees, both at the community level, i.e. for the forest as a whole, and individually for 87 species, which included 35 well-represented species ( $n \geq 20$ trees), 16 commercial species and 36 other species. Logistic regressions were used to determine the diameter (minimum and regular) of these species on fruiting. Reproductive phenology for the majority of the trees and the species is largely seasonal, annual and regular (81.6\%, 71 species). The peaks for flowering are more abrupt than the fruiting peaks and more spread out over time, although the timing of flowering and fruiting is significantly aggregated. Most of the trees and species bloom from December to February, during the short dry season, but flowers and fruits can be observed throughout the year within the community. Only 13 species showed a significant relationship between diameter and reproduction, including seven canopy species, five understory species and one light-demanding species. For these 13 species, the average minimum reproduction diameter was $17.3 \mathrm{~cm}$.

Keywords: tree size on reproduction, flowering, fruiting, semi-deciduous tropical moist forests, Mayombe forest, seasonality, phenological synchronicity, Democratic Republic of the Congo.

\section{RESUMEN}

Fuerte estacionalidad del clima y de la fenología reproductiva en el bosque de Mayombe: aporte de datos históricos de la Reserva de Luki en la República Democrática del Congo

En África, la fenología reproductiva de los árboles tropicales, mayoritariamente anual, parece presentar una cierta regularidad. Este estudio documenta las variaciones intra- e interanuales de la fenología reproductiva de los árboles del bosque de Mayombe a partir de los datos históricos de la Reserva de Luki (República Democrática del Congo). El diámetro de reproducción de las especies explotadas para obtener madera, todavía ampliamente desconocido, ha sido también examinado para las especies suficientemente representadas en los datos. El seguimiento fenológico de 3642 árboles pertenecientes a 158 especies y a 39 familias fue realizado cada 10 días desde 1948 hasta 1957. Las estadísticas circulares se utilizaron para probar el sincronismo de la fenología entre árboles, a escala de la comunidad, para el bosque en su conjunto, e individualmente para 87 especies: 35 especies bien representadas ( $\mathrm{n} \geq 20$ árboles), 16 especies comerciales y 36 otras especies. Mediante regresiones logísticas se pudo determinar el diámetro de fructificación (mínimo y regular) de estas especies. Para la mayoría de las especies, la floración era regular, anual y ampliamente estacional (81,6 \%, 71 especies). Los picos estaban más marcados para la floración que para la fructificación, más extendida en el tiempo, aunque con asociaciones temporales significativas. La mayor parte de los árboles y de las especies florecían entre diciembre y febrero, durante la corta estación seca, aunque se podían observar flores y frutos durante todo el año a escala de la comunidad. Solo 13 especies mostraron una relación significativa entre el diámetro y la reproducción, entre las cuales siete especies de dosel forestal, cinco de sotobosque y una heliófila. Para estas especies, la media del diámetro mínimo de reproducción resultó ser de 17,3cm.

Palabras clave: diámetro de reproducción, floración, fructificación, bosques tropicales húmedos, bosque de Mayombe, estacionalidad, sincronismo, República Democrática del Congo. 
La phénologie est l'étude de la périodicité des phénomènes biologiques récurrents. Dans le contexte actuel des changements globaux, et plus spécifiquement du changement du climat, des décalages phénologiques, ainsi que des modifications de la distribution des espèces ont déjà pu être observés (Walther et al., 2002 ; Chapman et al., 2005). Pour les arbres tropicaux, les processus les plus couramment étudiés sont la défeuillaison, la floraison, la fructification et la dissémination (stade où les fruits sont matures) (Lieth, 1974 ; Schwartz, 2003). La saisonnalité de l'activité cambiale et de la croissance bien qu'importante est néanmoins peu documentée (Beeckman, 2016). En effet, l'activité cambiale (et aussi la floraison) des arbres peut être inhibée par un stress hydrique qui se manifeste par la chute des feuilles (Borchert, 1999).

Dans les forêts tropicales, il est possible d'observer des arbres en fleurs et fruits toute l'année, bien qu'en proportions différentes (Sabatier et Puig, 1982). Différentes phénophases peuvent parfois être observées simultanément sur les arbres de la même espèce, et aussi sur les mêmes arbres (Whitmore, 1998). Un large éventail de stratégies phénologiques a par ailleurs été observé entre espèces coexistantes (Sakai, 2001 ; Bawa et al., 2003 ; Singh et Kushwaha, 2006). Newstrom et al. (1994) proposent une classification de la phénologie reprenant quatre groupes : phénologie continue (reproduction permanente avec parfois de brèves interruptions) ; phénologie sub-annuelle (plusieurs cycles de reproduction par an) ; phénologie annuelle (un cycle unique de reproduction par an) ; phénologie supra-annuelle (les cycles de reproduction dépassent une année). Les événements supra-annuels de fructification massive (mast event) ont été étudiés en Indonésie (Curran et Leighton, 2000), en Malaisie (Nagamitsu et Inoue, 2002), en Guyane française (Norden et al., 2007) et au Cameroun (Green et Newbery, 2002).

En Afrique, les arbres tropicaux semblent présenter une certaine régularité dans la floraison et la fructification. Une analyse multisites incluant des données d'Afrique de l'Ouest, d'Afrique centrale et d'Afrique de l'Est a notamment permis de mettre en évidence une dominance de la floraison et de la fructification annuelle pour l'ensemble des 12 sites étudiés (Adamescu et al., 2018). La dominance de la floraison et de la fructification annuelle au niveau de la communauté a également été identifiée par Chapman et al. (1999) en Ouganda, mais avec des variations en fonction des sites. La floraison et la fructification sont aussi fortement régulières et annuelles à Luki pour les espèces classées en groupes fonctionnels : espèces de canopée, espèces héliophiles et espèces de sousbois (Couralet et al., 2013). Une floraison annuelle a de même été mise en évidence pour la plupart des espèces suivies dans le Parc national de la Lopé, au Gabon (Bush et al., 2017), et pour des espèces étudiées individuellement, au Cameroun, Milicia excelsa (Daïnou et al., 2012), Baillonella toxisperma, Entandrophragma cylindricum et Erythrophleum suaveolens (Fétéké et al., 2016), et en RDC, Milettia laurentii (Menga et al., 2012). Malgré cette tendance, il existe néanmoins au sein des sites, entre et au sein des espèces, une diversité de comportements phénologiques dans plusieurs sites à travers l'Afrique tropicale (Bush et al., 2017 au Gabon ; Sun et al., 1996 au Rwanda ; Adamescu et al., 2018).
Outre la saisonnalité, la taille à la reproduction varie également entre les espèces d'arbres tropicaux, et au sein d'un site tous les arbres de la même espèce n'ont pas la même probabilité de fructifier (Plumptre, 1995 ; Wright et al., 2005). La taille à la reproduction n'est pas connue pour beaucoup d'espèces d'arbres (Thomas, 2011). Le diamètre de fructification régulière est pourtant une information essentielle pour la gestion et l'aménagement forestier (Durrieu de Madron et Daumerie, 2004 ; Plumptre, 1995). Dans la Réserve forestière de Budongo en Ouganda, Plumptre (1995) a montré que la probabilité de fructification augmentait en fonction du diamètre. Pour huit espèces exploitables en République centrafricaine, le diamètre de fructification régulière se situe entre $40 \mathrm{~cm}$ pour Mansonia altissima et $90 \mathrm{~cm}$ pour Triplochiton scleroxylon (Durrieu de Madron et Daumerie, 2004). En République démocratique du Congo (RDC), le diamètre de fructification régulière pour Millettia laurentii se situe entre 40 et $50 \mathrm{~cm}$, en fonction des sites (Menga et al., 2012). Pour laisser des arbres reproducteurs, des semenciers ou des porte-graines en nombre suffisant après le passage de l'exploitation, il faut s'assurer que le diamètre de reproduction régulière ou de reproduction efficace est inférieur au diamètre minimum à partir duquel les arbres sont exploités. Cela semble être le cas pour la plupart des espèces en Afrique centrale (Ouédraogo et al., 2018) même si les informations manquent encore pour les espèces typiquement exploitées en RDC comme Prioria balsamifera (Tola, Agba). Or, ceci permet d'orienter de manière objective un plan d'aménagement adapté à l'espèce, pour que son exploitation présente et future soit durable, laissant un certain nombre de semenciers après le passage de l'exploitation.

Dans cette étude, nous proposons de documenter la phénologie reproductive de la forêt du Mayombe à partir des données phénologiques et climatiques historiques de la Réserve de Luki. Des changements à long terme dans la phénologie ont déjà pu être mis en évidence en Afrique tropicale, notamment grâce aux 28 ans de suivi phénologique dans le Parc national de Kibale en Ouganda (Chapman et al., 2000). L'utilisation de données historiques permet d'établir un état et fonctionnement de référence, qui pourra servir de base pour le monitoring de la réponse au changement climatique. Les données phénologiques disponibles de 1948 à 1957 pour 3642 arbres, appartenant à 158 espèces, et les données climatiques locales disponibles sur la même période n'ont pour l'instant été utilisées que partiellement, en regroupant les espèces par groupes fonctionnels (Couralet et al., 2013). Nous proposons ici d'examiner la phénologie reproductive pour l'ensemble de la communauté et pour les espèces séparément. Plus spécifiquement, il s'agit : de déterminer le type de phénologie (continue, annuelle, sub-annuelle et supra-annuelle) de la communauté et des espèces selon l'approche graphique proposée par Newstrom et al. (1994); de tester le synchronisme de la floraison et de la fructification de la communauté et des espèces à l'aide des statistiques circulaires; d'examiner les variations intraet inter-annuelles de la phénologie reproductive en lien avec les précipitations ; de déterminer le diamètre de reproduction des espèces à partir de régressions logistiques. 


\section{Matériel et méthodes}

\section{Site de l'étude}

La Réserve de Luki est située au sud-ouest de la RDC (latitudes comprises entre $05^{\circ} 35^{\prime}$ et $05^{\circ} 4^{\prime}$ ' Sud, longitudes entre $13^{\circ} 10^{\prime}$ et $13^{\circ} 15^{\prime}$ Est), dans la province du Kongo central à $120 \mathrm{~km}$ de la côte atlantique. Elle constitue la pointe sud du massif forestier guinéo-congolais, à la limite entre le centre régional d'endémisme guinéo-congolais et la zone de transition régionale guinéo-congolaise/zambézienne (White, 1986). Elle est considérée comme représentative de la forêt du Mayombe (Lubini, 1997) et classée dans le type moist central Africa par Fayolle et al. (2014) correspondant à des forêts semi-décidues (White, 1986). Organisée en trois zones (zones centrale, de transition et tampon), elle couvre une superficie d'environ 33000 ha (figure 1) et est traversée sur sa quasi-totalité par le bassin hydrographique de la rivière Luki. Les sols sont généralement ferrallitiques, acides avec une faible teneur en cations (Sénéchal et al., 1989). Le climat est de type tropical humide $A w_{5}$ de la classification de Köppen (Peel et al., 2007), influencé par le courant marin froid de Bengwela et les alizés du sud-est (Olson et al., 2001), étant donné la proximité à la côte atlantique. La saison des pluies s'étale entre octobre et mai et la grande saison sèche entre juin et août, parfois septembre (Couralet et al., 2010, 2013). Une petite saison sèche est parfois perceptible entre décembre et février (Couralet et al., 2013).

Instituée par l'ordonnance n 05/AGRI du 12 janvier 1937 du gouverneur général de colonie du Congo belge, la Réserve de Luki faisait partie du domaine boisé de l'État et sa gestion fut confiée à l'Institut national pour l'étude agronomique du Congo belge (INEAC). Depuis l'indépendance, elle est gérée par l'Institut national pour l'étude et la recherche agronomiques (INERA) et est reconnue depuis 1979 comme réserve Man and Biosphere par l'Unesco.

\section{Des données phénologiques et climatiques historiques}

De 1947 à 1958, des observations phénologiques ont été réalisées tous les 10 jours sur 3642 arbres appartenant à 158 espèces, 123 genres et 39 familles botaniques. Les trois espèces les plus abondantes sont Prioria balsamifera (Fabaceae-Caesalpinioïdeae), $\mathrm{n}=194$ arbres, Terminalia superba (Combretaceae), $n=184$ arbres, et Funtumia elastica (Apocynaceae), $n$ $=149$ arbres. Parmi les 158 espèces suivies, 77 sont sempervirentes, 52 espèces sont décidues, et l'information est manquante pour 21 espèces parce que les observations sur le feuillage n'ont pas été faites (Couralet et al., 2013). Comme un faible nombre d'arbres a été suivi pendant la première année (1947, $n=652$, soit $17,9 \%)$ et la dernière année $(1958, n=179$, soit 4,9\%), ces deux années n'ont pas été intégrées dans les analyses. Toutefois, les données analysées ici couvrent 10 ans de suivi phénologique pour plus de 3000 arbres, et constituent un jeu de données unique pour les forêts d'Afrique centrale. À titre d'exemple, 856 arbres appartenant à 70 espèces et 26 familles sont suivis mensuellement depuis 1986 dans le Parc national de la Lopé au Gabon (Bush et al., 2017) et 3793 arbres appartenant à 104 espèces sont suivis mensuellement depuis 1990 dans le Parc national de Kibale en Ouganda (Chapman et al., 1999). Sur le terrain, les arbres ont été localisés le long de six sentiers dans la forêt de la Nkula, chaque arbre étant marqué avec un numéro unique inscrit sur une plaquette métallique (photo 2a). Les observations phénologiques étaient réalisées tous les dix jours par la même équipe et la présence/absence des différentes phénophases dans le houppier (défeuillaison, floraison, fructification et dissémination) notées de façon indépendante, les informations étant reportées dans des carnets phénologiques (photo 2b). La floraison et la fructification ont été notées lorsque les fleurs/fruits étaient observables sur au moins un quart du houppier. Pour tous les arbres, la mesure du diamètre pour le suivi de la croissance a été faite annuellement pendant la même période, de 1948 à 1957, et le diamètre initial de ces arbres est donc disponible.

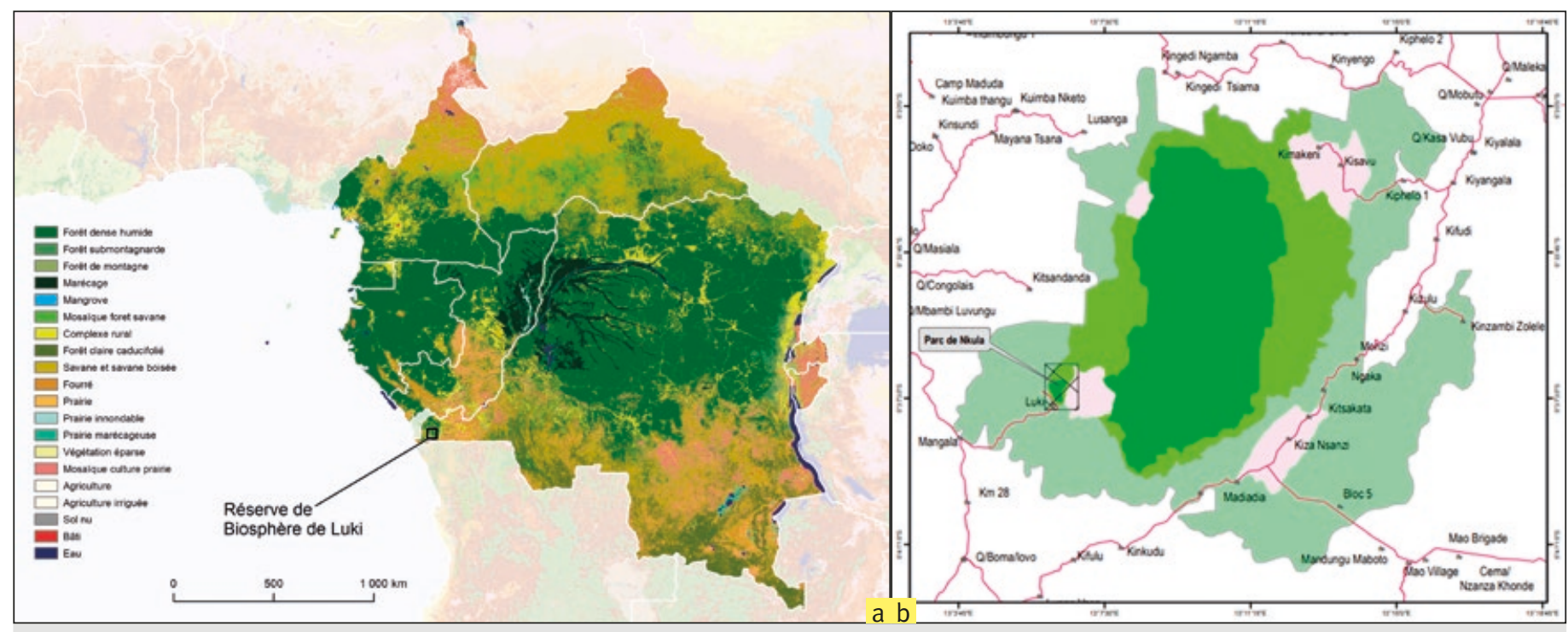

Figure 1.

Localisation de la Réserve de Luki : (a) les types de forêts et les classes d'occupation du sol rencontrés dans le bassin du Congo (Verhegghen et al., 2012) ; (b) les zones de la réserve et le parc phénologique de la Nkula.

Le vert foncé correspond à la zone centrale, et les verts plus clairs à la zone tampon et à la zone de transition. Les enclaves villageoises dans la réserve sont en rose clair, et les routes principales sont en rouge. 

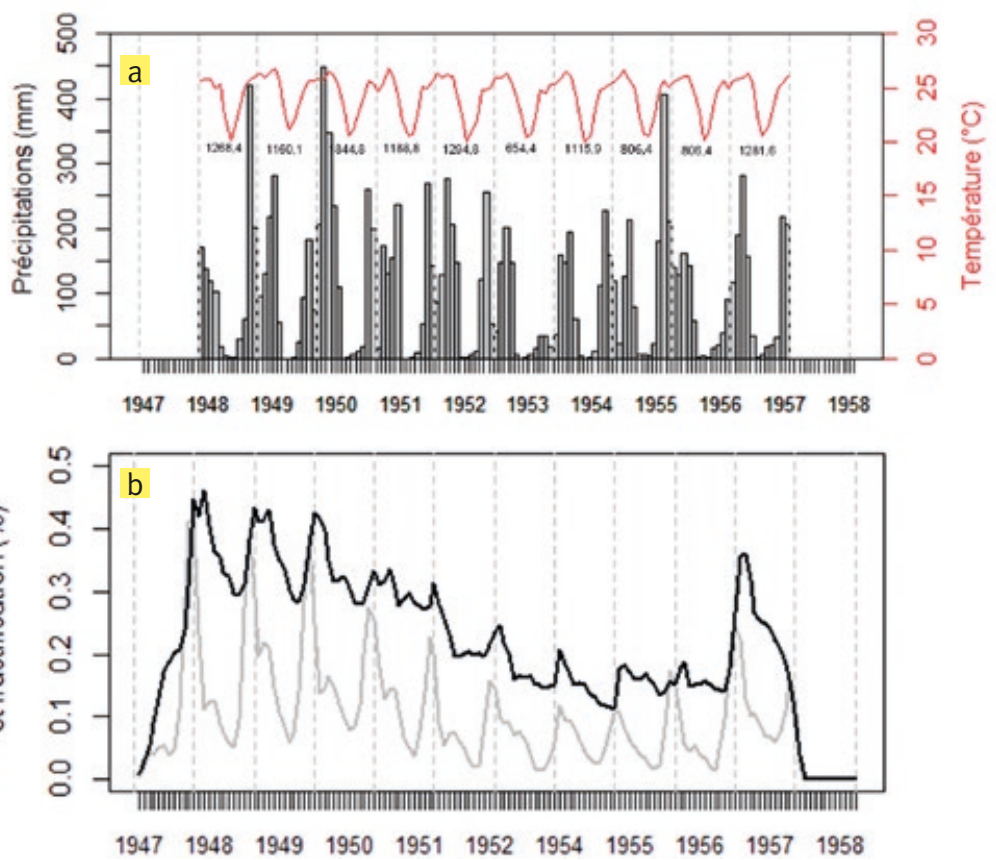

Figure 2.

Climat (a) et phénologie reproductive (b) issus des données historiques de la Réserve de Luki. La somme des précipitations mensuelles (barres grisées) avec des valeurs annuelles au-dessus et la moyenne des températures (courbe rouge) enregistrées par la station de Luki de 1948 à 1957 sont représentées. La proportion d'arbres observés en floraison (en gris) et en fructification (en noir) pour l'ensemble de la communauté (tous les arbres suivis quelle que soit l'espèce) et pour l'ensemble de la période d'observation, de 1947 à 1958, est donnée. Les lignes verticales grises discontinues indiquent les différentes années. certaines années une petite saison sèche. Par ailleurs, on observe une proportion d'arbres en fruits plus importante que la proportion d'arbres en fleurs (figure $2 \mathrm{~b}$ ), vraisemblablement parce que, depuis le sol, les fruits sont plus facilement observables que les fleurs dans les houppiers.

\section{Phénologie reproductive de la forêt du Mayombe}

La phénologie reproductive, floraison et fructification, dans la forêt du Mayombe apparaît annuelle et largement saisonnière (annexe 1).

À l'échelle de la communauté, un pic de floraison est bien identifiable chaque année (figure 2b), mais son intensité semble diminuer de 1951 à 1956. Ce pic de floraison en fin (décembre) ou début (janvier-février) d'année calendaire semble correspondre à la petite saison sèche (figure 2a). Les statistiques circulaires ont permis de révéler une floraison significativement synchrone entre les arbres au sein de la communauté (tableau I). La date du pic de floraison varie néanmoins entre les années (du 13 novembre au 13 mars). Et, si le pic de floraison est marqué chaque année, il existe tout au long de l'année des arbres en fleurs et portant des fruits dans la forêt du Mayombe (figure 2b).

Bien que significatif pour toutes les années d'observation, le synchronisme à l'échelle de la communauté est moins patent pour la fructification que pour la floraison (tableau I, rho $<0,14$ pour la fructification et $r h o>0,20$ pour la floraison) car la fructification est plus étalée dans le temps

\begin{tabular}{|c|c|c|c|c|c|c|}
\hline \multirow{3}{*}{ Année } & \multicolumn{6}{|c|}{$\begin{array}{l}\text { Tableau I. } \\
\text { Synchronisme des arbres en fleurs et portant des fruits à l'échelle de la communauté pour les dix années } \\
\text { de suivi phénologique dans la Réserve de Luki (1948-1957). Les résultats des statistiques circulaires } \\
\text { (rho et date) et la significativité }(P \text { d'après le test d'uniformité de la distribution de Rayleigh) sont donnés } \\
\text { pour la floraison et pour la fructification, et ce pour chaque année de suivi phénologique. }\end{array}$} \\
\hline & & Flora & & & Fructifi & \\
\hline & rho & $P$ & Date du pic & rho & $P$ & Date du pic \\
\hline 1948 & 0,35 & $<0,001$ & 07 décembre & 0,07 & $<0,001$ & 16 février \\
\hline 1949 & 0,30 & $<0,001$ & 13 décembre & 0,08 & $<0,001$ & 26 février \\
\hline 1950 & 0,21 & $<0,001$ & 10 décembre & 0,05 & $<0,001$ & 17 mars \\
\hline 1951 & 0,27 & $<0,001$ & 04 janvier & 0,02 & $<0,001$ & 22 avril \\
\hline 1952 & 0,29 & $<0,001$ & 26 décembre & 0,05 & $<0,001$ & 14 janvier \\
\hline 1953 & 0,37 & $<0,001$ & 13 mars & 0,05 & $<0,001$ & 11 février \\
\hline 1954 & 0,29 & $<0,001$ & 04 février & 0,08 & $<0,001$ & 29 mars \\
\hline 1955 & 0,42 & $<0,001$ & 25 décembre & 0,05 & $<0,001$ & 22 avril \\
\hline 1956 & 0,50 & $<0,001$ & 12 décembre & 0,06 & $<0,001$ & 04 janvier \\
\hline 1957 & 0,20 & $<0,001$ & 30 décembre & 0,14 & $<0,001$ & 09 avril \\
\hline
\end{tabular}


(figure 2b). Un pic de fructification est néanmoins identifiable à l'échelle de la communauté pour l'ensemble des années d'observation, avec une date variant entre début janvier et fin avril, et qui correspond pour la plupart des années à la saison des pluies. L'intensité de la fructification semble également plus faible de 1951 à 1956, en réponse à des années moins arrosées (figure 2a).

\section{Phénologie reproductive des espèces du Mayombe}

La phénologie reproductive (floraison et fructification) apparaît annuelle et largement saisonnière pour la majorité des espèces de la forêt du Mayombe.

Quand on considère l'ensemble des données, de 1948 à 1957, la floraison est significativement agrégée temporellement $(P<0,001)$ pour toutes les espèces (annexe 2). Le pic de floraison est observé entre novembre et février et correspond à la fin de la grande saison des pluies (novembre, $n=19$, soit $21,8 \%$ des espèces), à la petite saison sèche (de décembre à janvier, $n=24$, soit 27,6 \%), ou parfois encore au début de la petite saison des pluies (février, $n=9$, soit 10,3\%). Il existe de fortes variations entre les espèces, entre Chrysophyllum africanum (Sapotaceae), qui présente la floraison la plus ponctuelle $\left(r h o_{\text {esp }}=0,93\right)$, et Pseudospondias microcarpa (Anacardiaceae), qui présente la floraison la plus étalée et la moins synchrone $\left(r h o_{\text {esp }}=0,04\right)$ bien que significativement agrégée temporellement. Si la majorité des espèces sont annuelles ( $n=71$, soit $81,6 \%$ ), l'analyse graphique révèle néanmoins quelques espèces sub-annuelles ( $n=10$, soit $11,5 \%$ ) et supra-annuelles ( $n=6$, soit $6,9 \%$ ). Pour la plupart des espèces, la date du pic de floraison varie entre les années, et ces variations semblent plus importantes pour les espèces sub-annuelles et supra-annuelles que pour les espèces annuelles (figure 3). La majorité des épisodes de floraison ont eu lieu en fin et en début d'année, avec très peu d'épisodes de floraison en juin, juillet et août, pendant la grande saison sèche. En ce qui concerne la fructification, comme à l'échelle de la communauté, les statistiques circulaires mettent en évidence un plus faible synchronisme $\left(r h o_{e s p}<0,5\right)$ que pour la floraison (résultats non présentés) bien que des pics de fructification soient observables chaque année pour la majorité des espèces (annexe 2).

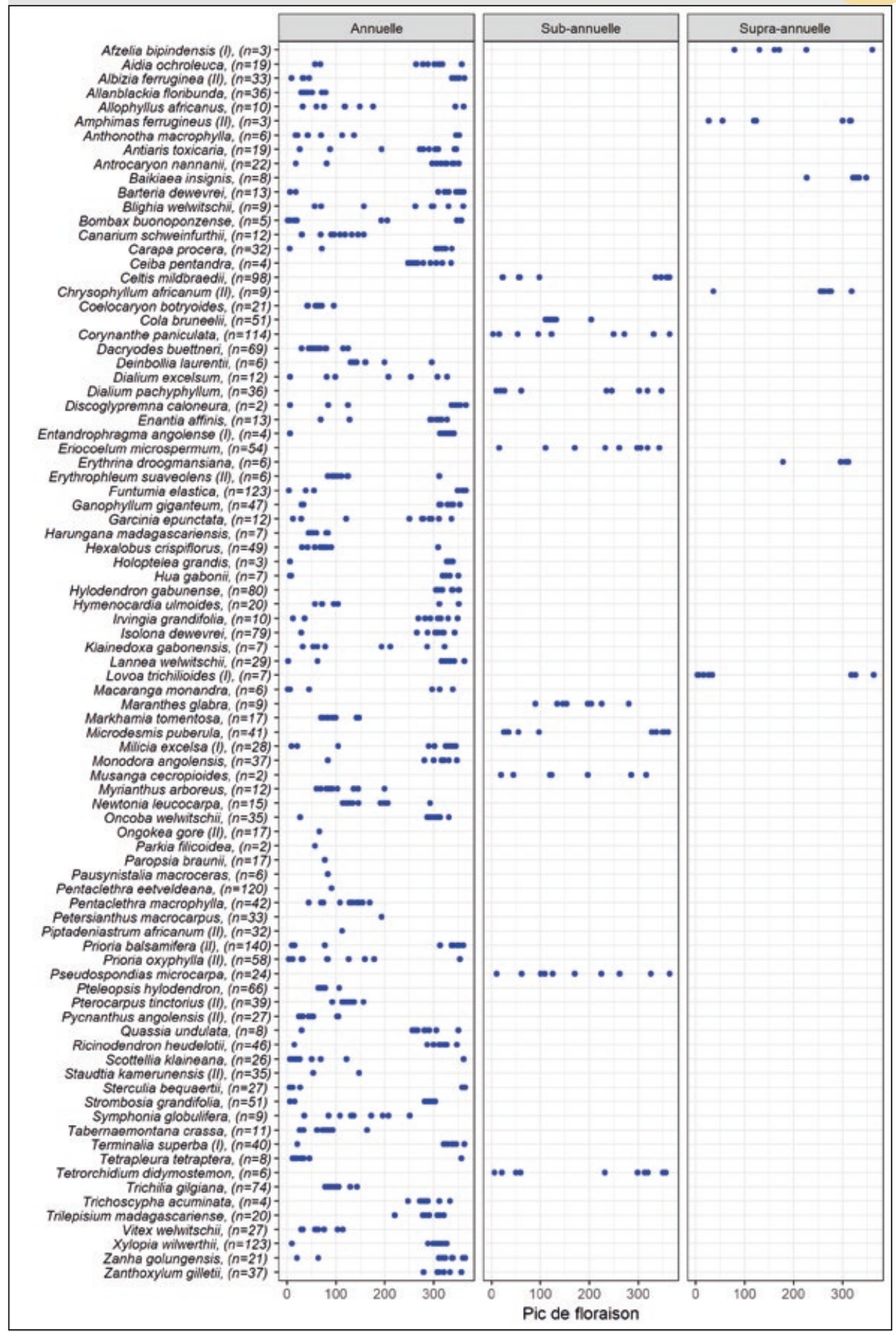

Figure 3.

Variation du pic de floraison des 87 espèces comprenant les espèces bien représentées $(n \geq 20)$, les espèces commerciales et d'autres espèces non commerciales et moins bien représentées. Les espèces commerciales sont indiquées par (I) et (II) en fonction de la classification en vigueur en RDC. Un point correspond à la valeur du pic (issue des statistiques circulaires) pour une année d'observation et pour une espèce donnée. Les espèces sont groupées en phénologie annuelle, sub-annuelle et supra-annuelle selon l'approche graphique de Newstrom et al. (1994). L'axe des abscisses représente les jours juliens, c'est-à-dire les 365 jours de l'année depuis le $1^{\text {er }}$ janvier. Le nombre d'arbres suivis est entre parenthèses. 


\section{Diamètre de reproduction des espèces du Mayombe}

Il existe une certaine variabilité du diamètre de reproduction entre les espèces suivies pour la phénologie dans la Réserve de Luki, mais la plupart des espèces commencent à fructifier à partir d'un faible diamètre $(4 \mathrm{~cm})$.

Pour les 13 espèces qui ont montré une relation significative entre le diamètre et le statut reproducteur, le diamètre de reproduction régulière théorique (point d'inflexion de la régression logistique) se situe entre $10 \mathrm{~cm}$ (Corynanthe paniculata, Rubiaceae ; Dacryodes buettneri, Burseraceae ; Funtumia elastica, Apocynaceae ; Xylopia wilwerthii, Annonaceae) et $44 \mathrm{~cm}$ (Eriocoelum microspermum, Sapindaceae ; annexe 2 et figure 4), avec une moyenne de $22,8 \mathrm{~cm}$. Les modèles n'ont pas pu être ajustés pour 47 espèces, soit parce que tous les arbres suivis n'étaient pas reproducteurs ( $\mathrm{n}=2$, soit 2,7 \%, Markhamia tomentosa, Bignoniaceae ; Microdesmis puberula, Pandaceae), soit parce que les arbres pouvaient être reproducteurs quel que fût leur diamètre $(n=45$, soit $60 \%$; annexe 2$)$. Pour certaines espèces, la probabilité de reproduction (i.e. fructification) en fonction du diamètre n'a pas pu être modélisée parce que le nombre d'arbres suivis était faible ( $\mathrm{n}=27$ espèces $<20$ arbres, soit $36,5 \%$; annexe 2 ). Le diamètre minimum de reproduction est également très variable entre les espèces (annexe 2 et figure 4), depuis $4 \mathrm{~cm}$ pour Microdesmis puberula jusqu'à $52 \mathrm{~cm}$ pour Symphonia globulifera (Clusiaceae), avec une moyenne de $28 \mathrm{~cm}$.

\section{Discussion}

\section{Une phénologie reproductive majoritairement annuelle}

Dans cette étude, nous avions pour objectif de caractériser la phénologie reproductive des arbres et espèces de la forêt du Mayombe sur la base du plus grand jeu de données disponible pour l'Afrique centrale (plus de 3000 arbres suivis tous les 10 jours pendant plus de 10 ans). Les données historiques de la Réserve de Luki montrent une forte saisonnalité à l'échelle de la communauté avec une majorité des arbres qui fleurissent en décembre, janvier et février. La phénologie reproductive, floraison et fructification, dans la forêt du Mayombe apparaît annuelle et largement saisonnière (annexe 2), démontrée ici, et précédemment par Couralet et al. (2013) pour des groupes fonctionnels, confirmant une tendance déjà observée en Ouganda (Chapman et al., 2005), au Gabon (Bush et al., 2017) et dans une étude multisites à travers l'Afrique tropicale (Adamescu et al., 2018). L'analyse de Fourier réalisée sur la phénologie de 856 arbres appartenant à 70 espèces et 26 familles dans le Parc national de la Lopé, au Gabon, a permis d'identifier une floraison annuelle sans un synchronisme apparent au niveau des espèces (Bush et al., 2017). Dans cette étude, les statistiques circulaires appliquées à 87 espèces de la forêt du Mayombe ont permis de mettre en évidence la floraison et la fructification annuelles et régulières, et un synchronisme à l'échelle de la communauté et au sein des espèces avec une floraison entre décembre et février. Aucune espèce à floraison strictement continue n’a été observée.

\section{Une synchronisation climatique et une saisonnalité " nord »}

Si la phénologie est régulière et majoritairement annuelle pour les arbres et les espèces de la forêt du Mayombe, la date et l'intensité des pics de floraison et de fructification varient néanmoins entre les années, comme identifié par certains auteurs à l'échelle de la communauté (Sabatier et Puig, 1982 dans la forêt dense guyanaise ; Sakai, 2001 pour les forêts tropicales) et à l'échelle des espèces (Newstrom et al., 1994 dans la forêt tropicale humide au Costa Rica ; Borchert, 1999 dans les forêts tropicales sèches en Amérique centrale et en Asie ; Singh et Kushwaha, 2006 dans une forêt tropicale décidue en Inde ; Wright et al., 1999, 2005 au Panama). Une plus faible proportion
Figure 4.

Régression logistique pour les 13 espèces d'arbres qui ont montré une relation significative entre le statut reproducteur et le diamètre (annexe 2). 
d'arbres en fleurs et/ou fruits a notamment pu être observée en réponse à des années moins arrosées. Cette baisse de l'intensité de la floraison et de la fructification entre 1951 et 1955 a déjà été mise en évidence par Couralet et al. (2013).

Les espèces du Mayombe fleurissent abondamment pendant la petite saison sèche, et le lien entre floraison et saison sèche a déjà été démontré à plusieurs reprises. Dieterlen (1978), en étudiant la phénologie dans la forêt équatoriale du Kivu en RDC, a constaté que plus de la moitié des espèces fleurissent durant la saison sèche et lorsque l'intensité des pluies reprend. Williams et al. (2008) ont montré que, dans la forêt tropicale saisonnière à l'ouest de la Thaïlande, la reproduction des arbres en forêts tropicales est dépendante des premières pluies et de la saison sèche. Pires O’Brien (1993) a décrit la floraison pendant la saison sèche en Amazonie. Sun et al. (1996) ont présenté des résultats similaires dans les forêts de montagne au Rwanda. La fructification irrégulière mais abondante pendant les saisons sèches a été rapportée par Sabatier et Puig (1982) et Couralet et al. (2013). L'absence de pic de floraison des espèces du Mayombe pendant la grande saison sèche pourrait s'expliquer par le fait que la grande saison sèche dans le Mayombe en RDC est caractérisée par une baisse légère de température et de fréquents brouillards matinaux ou brumes, qui compensent le déficit en eau du sol (Lubini, 1997 ; Couralet et al., 2013). L'influence de l'insolation et de la longueur du jour sur phénologie a été mise en évidence sous les tropiques (Calle et al., 2010 ; Borchert et al., 2015).

Grâce aux données historiques, nous avons pu mettre en évidence qu'une majorité des arbres et des espèces du Mayombe fleurissent entre décembre et février, pendant la petite saison sèche au sud de l'équateur climatique qui correspond à la grande saison sèche au nord de l'équateur. Il est intéressant de noter le synchronisme de la floraison pour la forêt du Mayombe, à l'extrême sud du bassin du Congo, avec d'autres études situées au nord de l'équateur climatique : au Costa Rica (Borchert, 1980), aux Philippines (Hamann, 2004), en Inde (Singh et Kushwaha, 2006).

\section{Une reproduction relativement précoce}

Une grande diversité des diamètres de reproduction a été mise en évidence au sein des espèces de la forêt du Mayombe, en accord avec les résultats de Wright et al. (2005) au Panama et pour certaines espèces exploitables en Afrique de l'Est (Plumptre, 1995) et dans le bassin du Congo (Durrieu de Madron et Daumerie, 2004 ; Menga et al., 2012 ; Ouédraogo et al., 2018). Le diamètre de reproduction régulière des 13 espèces qui ont montré une relation significative entre le statut reproducteur et le diamètre varie entre 10 et $44 \mathrm{~cm}$. Le plus petit diamètre de reproduction/fructification régulière $(10 \mathrm{~cm})$ est inférieur à celui de $30 \mathrm{~cm}$ (Chrysophyllum boukokoënse, Lophira alata) obtenu en République centrafricaine et au Cameroun (Ouédraogo et al., 2018) et de $40 \mathrm{~cm}$ (Mansonia altissima) obtenu en République centrafricaine (Durrieu de Madron et Daumerie, 2004), pour des espèces commerciales, majoritairement de la canopée. Les espèces suivies dans la forêt du Mayombe se reproduisent donc vraisemblablement à partir d'un petit diamètre, ce qui est intéressant pour la reconstitution de la forêt, même si la fructification régulière ne garantit pas la régénération naturelle. Les diamètres minimums de reproduction observés (à partir de $4 \mathrm{~cm}$ ) indiquent également que les espèces du Mayombe se reproduisent à partir de petits diamètres. Les espèces qui se reproduisent entre 4 et $7 \mathrm{~cm}$ sont principalement des espèces de sous-bois (cinq espèces sur sept).

La plupart des espèces $(n=47)$ n'ont pas montré de relation significative entre leur statut reproducteur et le diamètre. Pour la plupart de ces espèces, les arbres suivis étaient reproducteurs quel que fût leur diamètre, et pour seulement deux espèces, les arbres ne se sont pas ou se sont peu reproduits. En se référant à la classification des espèces de Couralet et al. (2013), parmi les 13 espèces qui ont montré une relation significative entre le diamètre et la reproduction, on trouve sept espèces de canopée (CAN), cinq de sous-bois (UND) et une héliophile (HEL) (annexe 2). Par contre, pour celles qui n'ont pas montré une relation significative entre le diamètre et la reproduction, on compte 41 espèces de canopée, 27 de sous-bois, cinq héliophiles et une espèce non classifiée.

\section{Conclusion}

Dans la forêt du Mayombe, une forte convergence des comportements phénologiques a pu être observée à partir des données historiques uniques disponibles pour la Réserve de Luki. Pour la majorité des arbres et des espèces, la phénologie reproductive est régulière, annuelle et largement saisonnière. La floraison semble calquée sur les précipitations, qui sont bimodales, avec deux saisons des pluies entrecoupées d'une grande saison sèche très marquée, et d'une petite saison sèche de moindre amplitude et intensité. La majorité des arbres et des espèces fleurissent entre décembre et février, pendant la petite saison sèche. Malgré ces tendances, la diversité des comportements phénologiques entre et au sein des espèces reste à explorer.

\section{Remerciements}

Les auteurs remercient les autorités de l'Institut national pour l'étude et la recherche agronomiques (INERA), de Gembloux Agro-Bio Tech, Université de Liège, et du Musée royal de l'Afrique centrale. Bhely Angoboy Ilondea bénéficie de l'appui du projet HERBAXYLAREDD (BR/143/ A3/HERBAXYLAREDD) pour le doctorat. 
Bois et Forêts des Tropiques - ISSN: L-0006-579X

48 Volume $341-3^{\text {rd }}$ quarter - July $2019-$ p. 39-53

\section{Annexe 1.}

Proportion d'arbres observés en floraison (en gris) et en fructification (en noir) pour chaque espèce bien représentée

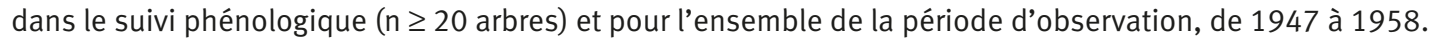
Les lignes verticales jaunes indiquent les différentes années.

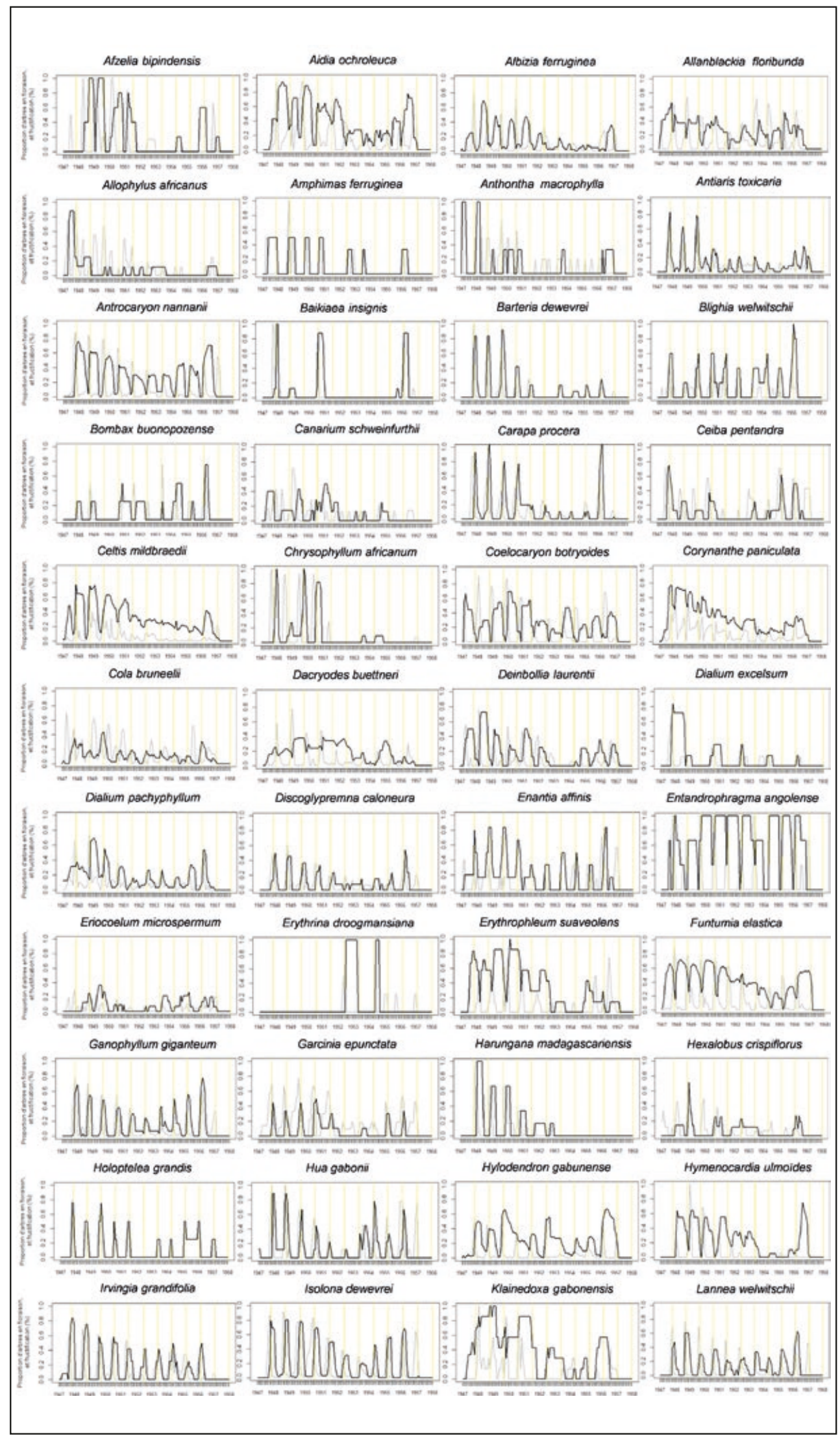


Annexe 1 (suite).

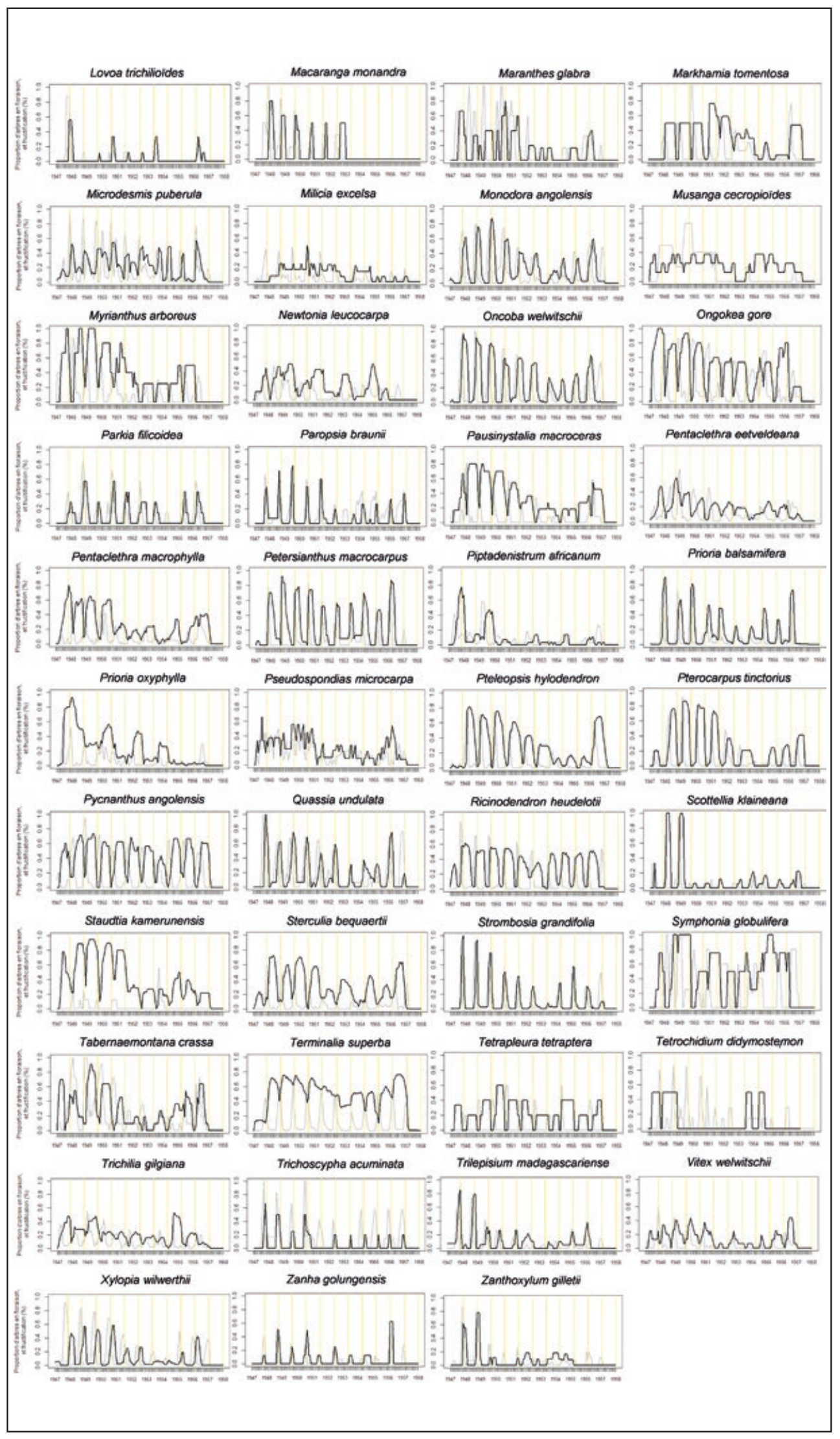


Bois et Forêts des Tropiques - ISSN: L-0006-579X

Volume 341 - $3^{\text {rd }}$ quarter - July 2019 - p. 39-53

OCUS / SEASONALITY AND REPRODUCTIVE PHENOLOGY

\section{Annexe 2.}

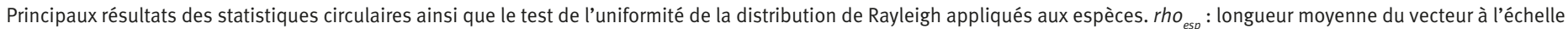

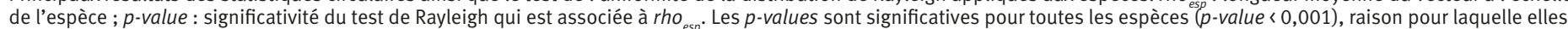

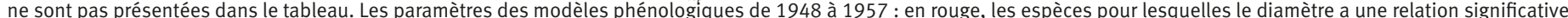

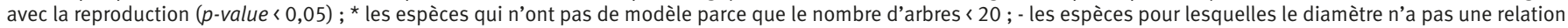

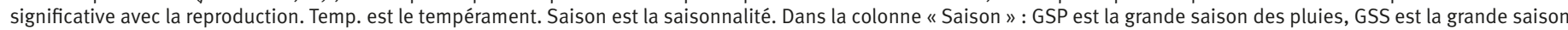

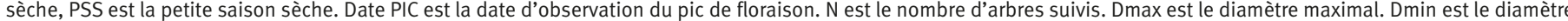

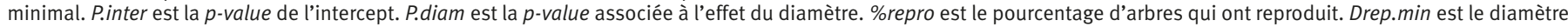
minimum de reproduction. Drep.thr est le diamètre de reproduction régulière.

\begin{tabular}{|c|c|c|c|c|c|c|c|c|c|c|c|c|c|c|c|c|}
\hline \multirow[t]{2}{*}{ Espèce } & \multirow[t]{2}{*}{ Famille } & \multirow[t]{2}{*}{ Temp. } & \multirow{2}{*}{$\begin{array}{l}\text { Classe de } \\
\text { fréquence }\end{array}$} & \multirow[b]{2}{*}{ Saison } & \multicolumn{4}{|c|}{ Statistiques circulaires } & \multirow[b]{2}{*}{ Dmin } & \multirow[b]{2}{*}{ Dmax } & \multirow[b]{2}{*}{ Intercept } & \multirow[b]{2}{*}{ P.inter } & \multicolumn{3}{|c|}{ Reproduction } & \multirow[b]{2}{*}{ Drep.thr } \\
\hline & & & & & Date PIC & $p$-value & $r h o_{\text {esp }}$ & $\mathbf{N}$ & & & & & P.diam & $\%$ repro & Drep.min & \\
\hline Antrocaryon nannanii De Wild. & Anacardiaceae & CAN & Annuelle & GSP & 23 mars & 0 & 0,41 & 22 & 23,9 & 128,6 & - & - & - & 91 & 24 & - \\
\hline Afzelia bipindensis Harms & $\begin{array}{l}\text { Fabaceae- } \\
\text { Caesalpinïodeae }\end{array}$ & COD & $\begin{array}{l}\text { Supra- } \\
\text { annuelle }\end{array}$ & GSP & 03 nov. & 0 & 0,39 & 3 & 22,8 & 28,8 & * & * & * & 33 & 28 & * \\
\hline $\begin{array}{l}\text { Aidia ochroleuca } \\
\text { (K. Schum.) E.M.A. Petit }\end{array}$ & Rubiaceae & UND & Annuelle & GSP & 29 avril & 0 & 0,16 & 19 & 9,9 & 110,4 & * & * & * & 95 & 10 & * \\
\hline $\begin{array}{l}\text { Albizia ferruginea. } \\
\text { (Guill. \& Perr.) Benth }\end{array}$ & $\begin{array}{l}\text { Fabaceae- } \\
\text { Mimosoïdeae }\end{array}$ & CAN & Annuelle & GSS & 03 sept. & 0 & 0,46 & 33 & 13,7 & 95,2 & $-6,67$ & 0,041 & 0,024 & 70 & 21 & $25[18 ; 31]$ \\
\hline Allanblackia floribunda Oliv. & Clusiaceae & COD & Annuelle & GSS & 19 juillet & 0 & 0,16 & 36 & 14 & 45,2 & - & - & - & 61 & 16 & - \\
\hline Allophylus africanus P. Beauv. & Sapindaceae & COD & Annuelle & GSS & 29 août & 0 & 0,4 & 10 & 23,2 & 58,9 & * & * & * & 80 & 23 & * \\
\hline $\begin{array}{l}\text { Amphimas ferrugineus } \\
\text { Pierre ex Pellegr. }\end{array}$ & $\begin{array}{l}\text { Fabaceae- } \\
\text { Faboïdeae }\end{array}$ & CAN & Annuelle & PSS & 01 février & 0 & 0,5 & 3 & 12,1 & 24,7 & * & * & * & 33 & 12 & * \\
\hline $\begin{array}{l}\text { Anthonotha macrophylla } \\
\text { P. Beauv. }\end{array}$ & $\begin{array}{l}\text { Fabaceae- } \\
\text { Caesalpinioïdeae }\end{array}$ & COD & Annuelle & GSP & 23 avril & 0 & 0,37 & 6 & 18,5 & 44,9 & * & * & * & 50 & 18 & * \\
\hline Antiaris toxicaria Lesch. & Moraceae & CAN & Annuelle & GSP & 16 déc. & 0 & 0,37 & 19 & 16,3 & 108,9 & * & * & * & 74 & 24 & * \\
\hline Baikiaea insignis Benth. & $\begin{array}{l}\text { Fabaceae- } \\
\text { Caesalpinioïdeae }\end{array}$ & COD & $\begin{array}{l}\text { Supra- } \\
\text { annuelle }\end{array}$ & PSS & 06 février & 0 & 0,76 & 8 & 20,2 & 30,6 & * & * & * & 88 & 22 & * \\
\hline $\begin{array}{l}\text { Barteria dewevrei } \\
\text { De Wild. \& T. Durand }\end{array}$ & Passifloraceae & COD & Annuelle & PSS & 17 janvier & 0 & 0,88 & 13 & 10,2 & 15,4 & * & * & * & 85 & 10 & * \\
\hline Blighia welwitschii (Hiern) Radlk. & Sapindaceae & CAN & Annuelle & GSP & 30 déc. & 0 & 0,64 & 9 & 24,5 & 51,6 & * & * & * & 56 & 26 & * \\
\hline Bombax buonopozense P. Beauv. & Bombacaceae & - & Annuelle & GSP & 05 mars & 0 & 0,43 & 5 & 25,1 & 51,8 & * & * & * & 60 & 26 & * \\
\hline Canarium schweinfurthii Engl. & Burseraceae & CAN & Annuelle & GSS & 22 août & 0 & 0,3 & 12 & 8,9 & 27 & * & * & * & 92 & 9 & * \\
\hline Carapa procera DC. & Meliaceae & UND & Annuelle & PSS & 25 janv. & 0 & 0,71 & 32 & 7,2 & 27 & - & - & - & 91 & 7 & - \\
\hline Ceiba pentandra (L.) Gaertn. & Bombacaceae & CAN & Annuelle & GSP & 27 déc. & 0 & 0,6 & 4 & 22,4 & 53,5 & * & * & * & 25 & 25 & * \\
\hline Celtis mildbraedii Engl. & Ulmaceae & CAN & $\begin{array}{c}\text { Sub- } \\
\text { annuelle }\end{array}$ & $\begin{array}{l}\text { GSP/ } \\
\text { PSS }\end{array}$ & 05 avril & 0 & 0,18 & 98 & 13,1 & 79,7 & $-9,02$ & 0,004 & 0,001 & 82 & 14 & $15[13 ; 17]$ \\
\hline Chrysophyllum africanum A. DC. & Sapotaceae & CAN & $\begin{array}{l}\text { Supra- } \\
\text { annuelle }\end{array}$ & PSS & 31 janv. & 0 & 0,72 & 9 & 10,5 & 21,6 & * & * & * & 67 & 10 & * \\
\hline $\begin{array}{l}\text { Coelocaryon botryoides } \\
\text { Vermoesen }\end{array}$ & Myristicaceae & CAN & Annuelle & GSS & 03 août & 0 & 0,35 & 21 & 9,1 & 19,1 & - & $\cdot$ & - & 62 & 9 & - \\
\hline Cola bruneelii De Wild. & Sterculiaceae & UND & Annuelle & PSS & 11 janv. & 0 & 0,31 & 51 & 6 & 67 & - & - & - & 71 & 6 & - \\
\hline Corynanthe paniculata Welw. & Rubiaceae & UND & $\begin{array}{l}\text { Sub- } \\
\text { annuelle }\end{array}$ & $\begin{array}{l}\text { GSS/ } \\
\text { GSS }\end{array}$ & 09 juillet & & 0,06 & 114 & 11,1 & 80,2 & $-5,07$ & 0,107 & 0,024 & 90 & 12 & $10[10 ; 11]$ \\
\hline $\begin{array}{l}\text { Dacryodes buettneri } \\
\text { (Engl.) H.J. Lam }\end{array}$ & Burseraceae & UND & $\begin{array}{l}\text { Sub- } \\
\text { annuelle }\end{array}$ & GSP & 16-sept. & 0 & 0,1 & 69 & 7,8 & 143,9 & 3,39 & 0,044 & 0,043 & 51 & 8 & $10[10 ; 10]$ \\
\hline Deinbollia laurentii De Wild. & Sapindaceae & - & Annuelle & GSP & 23 sept. & 0 & 0,49 & 6 & 16,6 & 75,8 & * & * & * & 67 & 17 & * \\
\hline Dialium excelsum Steyaert & $\begin{array}{l}\text { Fabaceae- } \\
\text { Caesalpinïodeae }\end{array}$ & CAN & Annuelle & PSS & 21 février & 0 & 0,21 & 12 & 12,7 & 65,6 & * & * & * & 50 & 22 & * \\
\hline Dialium pachyphyllum Harms & $\begin{array}{l}\text { Fabaceae- } \\
\text { Caesalpinïodeae }\end{array}$ & CAN & Annuelle & GSP & 11 mars & 0 & 0,37 & 36 & 11,1 & 142,3 & - & - & 0,688 & 64 & 11 & - \\
\hline $\begin{array}{l}\text { Discoglypremna } \\
\text { caloneura (Pax) Prain }\end{array}$ & Euphorbiaceae & - & Annuelle & PSS & 16 février & 0 & 0,65 & 2 & 17,8 & 46,7 & * & * & * & 50 & 18 & * \\
\hline Enantia affinis Exell & Annonaceae & UND & Annuelle & PSS & 06 janvier & 0 & 0,46 & 13 & 12,1 & 63,7 & * & * & * & 69 & 12 & * \\
\hline $\begin{array}{l}\text { Entandrophragma } \\
\text { angolense (Welw.) C. DC. }\end{array}$ & Meliaceae & CAN & Annuelle & GSP & 23 avril & 0 & 0,26 & 4 & 9,9 & 22,3 & * & * & * & 50 & 10 & * \\
\hline $\begin{array}{l}\text { Eriocoelum microspermum } \\
\text { Gilg ex Radlk. }\end{array}$ & Sapindaceae & CAN & Annuelle & GSP & 22 sept. & 0 & 0,05 & 54 & 8,8 & 90,9 & $-7,42$ & 0,001 & 0,004 & 28 & 14 & $44[37 ; 59]$ \\
\hline $\begin{array}{l}\text { Erythrina droogmansiana } \\
\text { De Wild. \& T. Durand }\end{array}$ & $\begin{array}{l}\text { Fabaceae- } \\
\text { Faboïdeae }\end{array}$ & UND & $\begin{array}{l}\text { Supra- } \\
\text { annuelle }\end{array}$ & GSP & 18 avril & 0 & 0,34 & 3 & 23,3 & 36,8 & * & * & * & 33 & 36 & * \\
\hline $\begin{array}{l}\text { Erythrophleum suaveolens } \\
\text { (Guill. \& Perr.) Brenan }\end{array}$ & $\begin{array}{l}\text { Fabaceae- } \\
\text { Caesalpinïodeae }\end{array}$ & CAN & Annuelle & GSP & 12 oct. & 0 & 0,21 & 6 & 22 & 32,9 & * & * & * & 67 & 22 & * \\
\hline $\begin{array}{l}\text { Funtumia elastica } \\
\text { (P. Preuss) Stapf }\end{array}$ & Apocynaceae & $\mathrm{PIO}$ & Annuelle & GSS & 18 juillet & 0 & 0,17 & 123 & 15,6 & 85 & $-4,4$ & 0,148 & 0,038 & 89 & 16 & $10[10 ; 14]$ \\
\hline $\begin{array}{l}\text { Ganophyllum giganteum } \\
\text { (A. Chev.) Hauman }\end{array}$ & Sapindaceae & CAN & Annuelle & PSS & 26 janvier & 0 & 0,67 & 47 & 4,8 & 122,9 & - & - & - & 66 & 15 & - \\
\hline Garcinia epunctata Stapf & Clusiaceae & UND & Annuelle & PSS & 12 janvier & 0 & 0,58 & 12 & 8,9 & 30 & * & * & * & 83 & 12 & * \\
\hline $\begin{array}{l}\text { Harungana madagascariensis } \\
\text { Lam. ex Poir. }\end{array}$ & Hypericaceae & $\mathrm{PIO}$ & Annuelle & GSS & 08 mai & 0 & 0,73 & 7 & 17,9 & 45,5 & * & * & * & 86 & 18 & * \\
\hline Hexalobus crispiflorus A. Rich. & Annonaceae & - & $\begin{array}{l}\text { Supra- } \\
\text { annuelle }\end{array}$ & GSP & 21 mars & 0 & 0,31 & 49 & 11,5 & 119,2 & $\cdot$ & - & - & 18 & 12 & - \\
\hline $\begin{array}{l}\text { Holoptelea grandis } \\
\text { (Hutch.) Mildbr. }\end{array}$ & Ulmaceae & CAN & Annuelle & GSP & 04 déc. & 0 & 0,67 & 3 & 25,1 & 29,2 & * & * & * & 100 & 25 & * \\
\hline Hua gabonii Pierre ex De Wild. & Huaceae & UND & Annuelle & PSS & 25 janvier & 0 & 0,56 & 7 & 11,8 & 47,1 & * & * & * & 100 & 12 & * \\
\hline Hylodendron gabunense Taub. & $\begin{array}{l}\text { Fabaceae- } \\
\text { Caesalpinioïdeae }\end{array}$ & CAN & Annuelle & PSS & 23 février & 0 & 0,25 & 80 & 8,9 & 60,5 & $-5,31$ & 0,077 & 0,025 & 82 & 14 & $11[10 ; 14]$ \\
\hline
\end{tabular}


Hymenocardia ulmoides Oliv.

Euphorbiaceae

PIO Annuelle

Irvingia grandifolia (Engl.) Engl. Irvingiaceae

CAN Annuelle

GSS 25 juillet

0 0,31

N Dmin Dmax

Intercept P.inter P.diam \% repro

Drep.min Drep.thr

Isolona dewevrei (De Wild.

\& T. Durand) Engl. \& Diels

Klainedoxa gabonensis

Annonaceae

UND Annuelle

Pierre ex Engl.

Lannea welwitschii (Hiern) Engl.

Irvingiaceae

CAN Annuelle

PSS 02 janvier

\begin{tabular}{l|l|l|l}
0,31 & 20 & 6,4 & 26,9 \\
\hline
\end{tabular}

Lovoa trichilioides Harms Meliacea

Macaranga monandra Müll. Arg. Euphorbiaceae

GSP 16 déc.

\begin{tabular}{llllll}
0 & 0,74 & 10 & 9,9 & 30,2 \\
\hline 0 & 0,75 & 79 & 6,8 & 28,4 \\
\hline
\end{tabular}

Maranthes glabra (Oliv.) Prance Chrysobalanaceae annuelle

Markhamia tomentosa (Benth.) Bignoniaceae

K. Schum. ex Engl.

Microdesmis puberula

Pandaceae

Hook. f. ex Planch.

Milicia excelsa (Welw.) C.C. Berg Moraceae

Monodora angolensis Welw. Annonaceae

Musanga cecropioides R. Br. Moraceae

Myrianthus arboreus P. Beauv.

Newtonia leucocarpa (Harms)

G.C.C. Gilbert \& Boutique

Oncoba welwitschii Oliv.

Ongokea gore (Hua) Pierre

Moraceae

Fabaceae-

Mimosoïdeae

Olacaceae

Parkia filicoidea Welw. ex Oliv. Fabaceae-

\begin{tabular}{ll} 
& Mimosoïdeae \\
\hline Paropsia braunii Gilg & Passifloraceae
\end{tabular}

UND Annuelle

Pausinystalia macroceras

UND

Annuelle

(K. Schum.) Pierre

Pentaclethra eetveldeana

De Wild. \& T. Durand

Rubiaceae

CAN

Sub-

annuelle

06 février

\begin{tabular}{|l|l|l|l|l|}
\hline 0,49 & 0,03 & 7 & 22,9 & 67,5 \\
\hline
\end{tabular}

0,2

-

85
$* \quad 80$

Pentaclethra macrophylla Faceae-

Benth.

Mimosoïdeae

Persianthus macrocarpus

Lecythidaceae

CAN Annuelle

(P. Beauv.) Liben

Piptadeniastrum africanum

Fabaceae-

Mimosoïdeae

Prioria balsamifera

(Vermoesen) Breteler

Prioria oxyphylla (Harms)

Breteler

Fabaceae-

Caesalpinioïdeae

Pseudospondias microcarpa Anacardiaceae

(A. Rich.) Engl.

Pteleopsis hylodendron Mildbr.

$\begin{array}{ll}\text { Pterocarpus tinctorius Welw. } & \text { Fabaceae- } \\ \text { Faboïdeae }\end{array}$

Pycnanthus angolensis

(Welw.) Warb.

Myristicaceae

Quassia undulata (Guill.

\& Perr.)D. Dietr.

Ricinodendron heudelotii

(Baill.) Pierre ex Heckel

Scottellia klaineana Pierre

Staudtia kamerunensis Warb.

PIO Subannuelle

PIO Annuelle

GSP 03 mars

GSP 12 nov.

$0 \quad 0,69$

\begin{tabular}{|l|l|l|l}
\hline 69 & 29 & 11,6 & 123,7
\end{tabular}

3,7

2,47

$0,2160,345$

$57 \quad 37$

$+x$ 


\section{Références bibliographiques}

Adamescu G. S., Plumptre A. J., Abernethy K. A., Polansky L., Bush E. R., Chapman C. A., et al., 2018. Annual cycles are the most common reproductive strategy in African tropical tree communities. Biotropica, 50 (3): 418-430. https://doi.org/10.1111/btp.12561

Bawa K. S., Kang H., Grayum M. H., 2003. Relationships among time, frequency, and duration of flowering in tropical rain forest trees. American Journal of Botany, 90: 877-887. https://doi.org/10.3732/ ajb.90.6.877

Beeckman H., 2016. Wood anatomy and trait-based ecology. IAWAJournal, 37 (2): 127-151. https://doi.org/10.1163/22941932-20160127

Borchert R., 1999. Climatic periodicity, phenology, and cambium activity in tropical dry forest trees. IAWA Journal, 20 (3): 239-247. https://doi.org/10.1163/22941932-90000687

Borchert R., Calle Z., Strahler A. H., Baertschi A., Magill R. E., Broadhead J. S., et al., 2015. Insolation and photoperiodic control of tree development near the equator. New Phytologist, 205: 7-13. https:// doi.org/10.1111/nph.12981

Borchert R., 1980. Phenology and ecophysiology of tropical trees: Erythrina poeppigiana. Ecology, 61: 1065-1074. https://doi. org/10.2307/1936825

Bush E. R., Abernethy K. A., Jeffery K., Tutin C., White L., Dimoto E., et al., 2017. Fourier analysis to detect phenological cycles using long-term tropical field data and simulations. Methods in Ecology and Evolution, 8 (5): 530-540. https://doi. org/10.1111/2041-210X.12704

Calle Z., Schlumpberger B. O., Piedrahita L., Leftin A., Hammer S. A., Tye A., et al., 2010. Seasonal variation in daily insolation induces bud break and flowering in the tropics. Trees, 24: 865-877. https:// doi.org/10.1007/s00468-010-0456-3

Chapman C., Wrangham R. W., Chapman L. J., Kennard D. K., Zanne A. E., 1999. Fruit and flower phenology at two sites in Kibale National Park, Uganda. Journal of Tropical Ecology, 15: 189-211. www.jstor. org/stable/2560018

Chapman C. A., Balcomb S. R., Gillespie T. R., Skorupa J. P., Struhsaker T. T., 2000. Long-term effects of logging on african primate communities: a 28-year comparison from Kibale national park, Uganda. Conservation Biology, 14: 207-217. https://doi. org/10.1046/i.1523-1739.2000.98592.x

Chapman C. A., Chapman L. J., Struhsaker T. T., Zanne A. E., Clark C. J., Poulson J. R., 2005. A long-term evaluation of fruiting phenology: importance of climate change. Journal of Tropical Ecology, 21: 31-45. https://doi.org/10.1017/S0266467404001993

Couralet C., Sterck F. J., Sass-Klaassen U., Van Acker J., Beeckman H., 2010. Species-specific growth responses to climate variations in understory trees of a Central African rain forest. Biotropica, 42: 503511. https://doi.org/10.1111/i.1744-7429.2009.00613.x

Couralet C., Van Den Bulcke J., Ngoma L. M., Van Acker J., Beeckman H., 2013. Phenology in functional groups of Central African rainforest trees. Journal of Tropical Forest Science, 25 (3): 361-374. https://www.jstor.org/stable/23617238

Curran L. M., Leighton M., 2000. Vertebrate responses to spatiotemporal variation in seed production of mast-fruiting Dipterocarpaceae. Ecological Monographs, 70: 101-128. https://doi.org/10.18 90/0012-9615(2000)070[0101:VRTSVI]2.0.CO;2
Daïnou K., Laurenty E., Mahy G., Hardy O. J., Brostaux Y., Tagg N., et al., 2012. Phenological patterns in a natural population of a tropical timber tree species, Milicia excelsa (Moraceae): Evidence of isolation by time and its interaction with feeding strategies of dispersers. American Journal of Botany, 99: 1453-1463. https://doi. org/10.3732/ajb.1200147

Davies S. J., Ashton P. S., 1999. Phenology and fecundity in 11 sympatric pioneer species of Macaranga (Euphorbiaceae) in Borneo. American Journal of Botany, 86 (12): 1786-1795. https://doi. org $/ 10.2307 / 2656675$

Dieterlen F., 1978. Zur Phänologie des Äquatorialen Regenwaldes im Ost-Zaire (Kivu): nebst Pflanzenliste und Klimadaten. Dissertationes botanicae, 47, J. Cramer, $111 \mathrm{p}$.

Durrieu de Madron L., Daumerie A., 2004. Diamètre de fructification de quelques essences en forêt naturelle centrafricaine. Bois et Forêts des Tropiques, 281 (3) : 87-95. http://revues.cirad.fr/index. $\mathrm{php/BFT/article/view/20230}$

Fayolle A., Swaine M. D., Bastin J., Bourland N., Comiskey J. A., Dauby G., et al., 2014. Patterns of tree species composition across tropical African forests. Journal of Biogeography, 41: 2320-2331. https://doi.org/10.1111/jbi.12382

Fétéké F., Fayolle A., Dainou K., Bourland N., Dié A., Lejeune P., et al., 2016. Variations saisonnières de la croissance diamétrique et des phénologies foliaire et reproductive de trois espèces ligneuses commerciales d'Afrique centrale. Bois et Forêts des Tropiques, 330 (4) : 3-21. https://doi.org/10.19182/bft2016.330.a31315

Green J. J., Newbery D. M., 2002. Reproductive investment and seedling survival of the mast-fruiting rain forest tree, Microberlinia bisulcata A. Chev. Plant Ecology, 162: 169-183. https://doi. org/10.1023/A:102030421

Hamann A., 2004. Flowering and fruiting phenology of a Philippine submontane rain forest: climatic factors as proximate and ultimate causes. Journal of Ecology, 92: 24-31. https://doi. org/10.1111/j.1365-2745.2004.00845.x

Lieth H., 1974. Purposes of a Phenology Book. In: Lieth H. (ed.). Phenology and Seasonality Modeling. Berlin, Heidelberg, Germany, Springer, Ecological Studies (Analysis and Synthesis), vol. 8, 3-19. https://doi.org/10.1007/978-3-642-51863-8 1

Lubini A., 1997. La végétation de la Réserve de Biosphère de Luki au Mayombe (RD Congo). Opera Botanica Belgica, 10, 155 p.

Menga P., Bayol N., Nasi R., Fayolle A., 2012. Phénologie et diamètre de fructification du wengé, Millettia laurentii De Wild. : implications pour la gestion. Bois et Forêts des Tropiques, 312 (2) : 31-41. https://doi.org/10.19182/bft2012.312.a20501

Morellato L. P. C., Talora C. D., Takahasi A., Bencke C. C., Romera C. E. E., Zipparo V., 2000. Phenology of atlantic rain forest trees: a comparative study. Biotropica, 32 (4b): 811-823. https://doi. org/10.1111/i.1744-7429.2000.tb00620.x

Nagamitsu T., Inoue T., 2002. Foraging activity and pollen diets of subterranean stingless bee colonies in response to general flowering in Sarawak, Malaysia. Apidologie, 33: 303-314. https://doi. org/10.1051/apido:2002016

Newstrom L. E., Frankie G. W., Baker H. G., 1994. A new classification for plant phenology based on flowering patterns in lowland tropical rain forest trees at La Selva, Costa Rica. Biotropica, 26: 141159. https://www.jstor.org/stable/2388804

Norden N., Chave J., Belbenoit P., Caubère A., Châtelet P., Forget P.-M., et al., 2007. Mast fruiting is a frequent strategy in woody species of Eastern South America. PLoS ONE, 2 (10): e1079. https:// doi.org/10.1371/journal.pone.0001079 
Olson D. M., Dinerstein E., Wikramanayake E. D., Burgess N. D., Powell G. V. N., Underwood E. C., et al., 2001. Terrestrial ecoregions of the World: a new map of life on Earth: A new global map of terrestrial ecoregions provides an innovative tool for conserving biodiversity. Bioscience, 51 (11): 933-938. https://doi. org/10.1641/0006-3568(2001)051[0933:TEOTWA]2.0.CO;2

Ouédraogo D.-Y., Doucet J.-L., Daïnou K., Baya F., Biwolé A. B., Bourland N., et al., 2018. The size at reproduction of canopy tree species in central Africa. Biotropica, 50 (3): 465-476. https://doi. org/10.1111/btp.12531

Peel M. C., Finlayson B. L., Mcmahon T. A., 2007. Updated world map of the Köppen-Geiger climate classification. Hydrology and Earth System Sciences, 11: 1633-1644. https://doi.org/10.5194/ hess-11-1633-2007

Pires O’Brien M. J., 1993. Phenology of tropical trees from Jari, Lower Amazon. I. Phenology of Amazon forest communities. Boletim do Museu Paraense Emílio Goeldi, 9 (1): 67-92.

Plumptre A. J., 1995. The importance of "seed trees" for the natural regeneration of selectively logged tropical forest. Commonwealth Forestry Review, 74 (3): 253-258. https://www.jstor.org/ stable/42608376

R Core Team, 2018. R: A language and environment for statistical computing. Vienna, Austria, R Foundation for Statistical Computing. https://www.R-project.org/

Sabatier D., Puig H., 1982. Phénologie et saisonnalité de la floraison et de la fructification en forêt dense guyanaise. Mémoires du Muséum National d'Histoire Naturelle, série A, Zoologie, tome 132, 173-184. http://horizon.documentation.ird.fr/exl-doc/pleins textes/pleins textes 5/b fdi 18-19/25170.pdf

Sakai S., 2001. Phenological diversity in tropical forests. Population Ecology, 43: 77-86. https://doi.org/10.1007/PL00012018

Schwartz M. D. (ed.), 2003. Phenology: An Integrative Environmental Science. Springer Netherlands, 564 p. https://link.springer. com/book/10.1007\%2F978-94-007-6925-0

Sénéchal J., Kabala M., Fournier F., 1989. Revue des connaissances sur le Mayombe. Paris, France, Unesco. http://dmcarc.com/ revue-des-connaissances-sur-le-mayombe-1989-pnud-unesco/

Singh K. P., Kushwaha C. P., 2006. Diversity of flowering and fruiting phenology of trees in a tropical deciduous forest in India. Annals of Botany, 97 (2): 265-276. https://doi.org/10.1093/aob/mcj028

Sun C., Kaplin B. A., Kristensen K. A., Munyaligoga V., Mvukiyumwami J., Ka Kajondo K., et al., 1996. Tree phenology in a tropical montane forest in Rwanda. Biotropica, 28 (4): 668-681. https:// www.jstor.org/stable/2389053

Thomas S. C., 2011. Age-related changes in tree growth and functional biology: The role of reproduction. In: Meinzer F. C., Lachenbruch B., Dawson T. E. (eds). Size- and age-related changes in tree structure and function. Springer, coll. Tree Physiology, 33-64. https://doi.org/10.1007/978-94-007-1242-3_2

Thomas S. C., 1996. Relative size at onset of maturity in rain forest trees: A comparative analysis of 37 Malaysian species. Oikos, 76: 145-154. https://www.researchgate.net/publication/262837830

Verhegghen A., Mayaux P., de Wasseige C., Defourny P., 2012. Mapping Congo Basin vegetation types from $300 \mathrm{~m}$ and $1 \mathrm{~km}$ multi-sensor time series for carbon stocks and forest areas estimation. Biogeosciences, 9 (12): 5061-5079. https://doi:10.5194/ bg-9-5061-2012

Walther G. R., Post E., Convey P., Menzel A., Parmesan C., Beebee T. J. C., et al., 2002. Ecological responses to recent climate change. Nature, 416: 389-395. https://doi.org/10.1038/416389a
White F., 1986. La végétation de l'Afrique - Mémoire accompagnant la carte de végétation de l'Afrique Unesco/AETFAT/UNSO. Paris, France, Orstom, Unesco, 385 p. http://horizon.documentation.ird. fr/exl-doc/pleins textes/divers11-06/24837.pdf

Whitmore T. C., 1998. Potential impact of climatic change on tropical rain forest seedlings and forest regeneration. Climatic Change, 39 (2-3): 429-438. https://doi.org/10.1023/A:1005356906898

Williams L. J., Bunyavejchewin S., Baker P. J., 2008. Deciduousness in a seasonal tropical forest in western Thailand: interannual and intraspecific variation in timing, duration and environmental cues. Ecologia, 155: 571-582. https://doi.org/10.1007/ s00442-007-0938-1

Wright S. J, Jaramillo M., Pavon J., Condit R., Hubbell S., Foster R., 2005. Reproductive size thresholds in tropical trees: Variation among individuals, species and forests. Journal of Tropical Ecology, 21 (3): 307-315. https://doi.org/10.1017/S0266467405002294

Wright S. J., Carrasco C., Calderón O., Paton S., 1999. The El Niño southern oscillation, variable fruit production, and famine in a tropical forest. Ecology, 80: 1632-1647. https://doi. org/10.1890/0012-9658(1999)080[1632:TENOSO]2.0.CO;2

\section{Angoboy Ilondea et al. - Contribution des auteurs}

\begin{tabular}{|c|c|}
\hline Rôle du contributeur & Noms des auteurs \\
\hline Conceptualisation & $\begin{array}{l}\text { B. Angoboy Ilondea, H. Beeckman, } \\
\text { D.-Y. Ouédraogo, N. Bourland, T. De } \\
\text { Mil, J. Van Den Bulcke, J. Van Acker, } \\
\text { C. Couralet, C. Ewango, W. Hubau, } \\
\text { B. Toirambe, J.-L. Doucet, A. Fayolle }\end{array}$ \\
\hline Gestion des données & $\begin{array}{l}\text { B. Angoboy llondea, H. Beeckman, } \\
\text { J. Van Den Bulcke, J. Van Acker, } \\
\text { C. Couralet, A. Fayolle }\end{array}$ \\
\hline Analyse formelle & $\begin{array}{l}\text { B. Angoboy llondea, H. Beeckman, } \\
\text { D.-Y. Ouédraogo, T. De Mil, A. Fayolle }\end{array}$ \\
\hline Méthodologie & $\begin{array}{l}\text { B. Angoboy llondea, H. Beeckman, } \\
\text { D.-Y. Ouédraogo, C. Ewango, } \\
\text { W. Hubau, J.-L. Doucet, A. Fayolle }\end{array}$ \\
\hline $\begin{array}{l}\text { Écriture - Préparation } \\
\text { de l'ébauche originale }\end{array}$ & $\begin{array}{l}\text { B. Angoboy Ilondea, H. Beeckman, } \\
\text { D.-Y. Ouédraogo, T. De Mil, A. Fayolle }\end{array}$ \\
\hline $\begin{array}{l}\text { Écriture - Révision } \\
\text { et édition }\end{array}$ & $\begin{array}{l}\text { H. Beeckman, D.-Y. Ouédraogo, } \\
\text { N. Bourland, J. Van Den Bulcke, } \\
\text { J. Van Acker, C. Couralet, B. Toirambe, } \\
\text { A. Fayolle }\end{array}$ \\
\hline
\end{tabular}

Bois et Forêts des Tropiques - Revue scientifique du Cirad
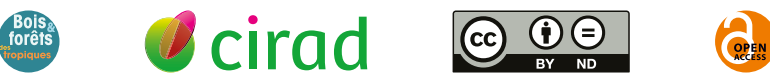

Cirad - Campus international de Baillarguet, 34398 Montpellier Cedex 5, France - Contact : bft@cirad.fr - ISSN : L-0006-579X 